\title{
IMPROVED ONTOLOGY MODEL FOR GATHERING WEB INFORMATION
}

\author{
Sayali Dharmendra Behere', R. B. Wagh ${ }^{2}$ \\ ${ }^{I}$ PG Student, Department of Computer Engineering, C. Patel Institute of Technology Shirpur, Maharashtra, India \\ ${ }^{2}$ Associate Professor, Department of Computer Engineering R. C. Patel Institute of Technology Shirpur, Maharashtra, \\ India
}

\begin{abstract}
A mode which is for purpose of discription knowledge also method of formalization, these various ontology profile are commonly used for representing most of user profiles in a manner of personalization form of web gathering information. Representing these profiles of various user's, most of these models shares their knowledge only from a global knowledge base which also called as local information of a user. In a paper, personalized ontology model gives information about for knowledge representation and various reasons for profiles of user. Ontology model gain knowledge of ontological profiles of a user from world knowledge base as well as local instance repositories of a user. This ontology model is also developed by differentiating to other models so in this way trec model, category model also a web model. In this way the results shows or gives the information to us that how this ontology model is successful than these benchmark models.
\end{abstract}

Keywords: Ontology, local instance repository, user profiles, web information gathering.

\section{INTRODUCTION}

There is various kind of information related to information of web is present and also it is being rapidly incremented. Achieving this useful information obtaining from web has challenging part of issue or problems for most of the users. Currently added web related information gathered attempted system or gain to each $n$ every user's prerequisites, which has been providing by user's information needs. For this type of reason user also will create his profiles which are already created for user background description of knowledge [1].

To represent the user profiles namely the concept models which is given by various user's when web gathering information process is run. This concept model is also given implicitly by user's and it also generated from user's background knowledge. Many web ontological user's have been observed in user's behavior or performance. Whenever user's read throughout a whole documentation. User's also determine easily is it of their user's convenience to them. Concept model which can be simulated by user's and representation of user profiles can created[2].

Simulation of user concept models are widely distributed inontology. Knowledge description models are utilized in personalizedweb information manner to gathering some web relatedinformation. These ontology models are called ontological userprofiles. Representing user profiles, many user's have research attempting to discover background knowledge of user's through local analysis [3].

Concept of global analysis for knowledge bases uses for user's knowledge of background. Knowledge concept base which commonly involves ontology profiles and gives also various knowledge bases. This type of global analysis technique also produce effective or efficient performance for background knowledge of user's [4].

Local concept of analysis gives local information of user's and it also observe performance of user's into ontological user's profiles. Some of the ontological profiles groups gives training for ontology profiles repeatedly from profiles user browse cache information. Background knowledge of user have been discover for user's feedback. Techniques of local analysis also depends upon data mining. These are techniques of classification for knowledge discovery [5].

The concept of world knowledge base and Local Instance Repository(LIR) of user's are also included in this ontology model. Concept of Local Instance Repository is personal collection of user's for providing information related to items. The model is also developed by differentiate these various by using a huge amount of standardized data set. These shows that this model of ontology is very successful [6]. In this paper, this ontology model simulates user's concept models by using this ontology personalized model which gives related information and it attempts to give improve web information which is achieving or gathering a performance of user's by using ontological user profiles for gathering or achieving web related information[7].

\subsection{The Concept of Ontology Model}

Model of Ontology very important nature study of being existing, as well as also the basic classification of user's categories and also of relationship concepts. Model for description of knowledge and also for formalization these ontology concepts are mostly representing these user profiles in the form of personalized web information 
gathering. An ontology model is also defined as a set or bunch of representational primitives or attributes with which for a model of domain knowledge. The representational attributes are mostly classes, attributes and relationships. The definition of the representational primitives include information for their meaning and rules which are logically consistent application. In our computer science model of ontology represents knowledge as a set of concepts within the domain and also the relationships between of those concepts. It also can be used to reasoning about the entities which are within that domain and also it may be used to describe these domain [5].

\subsection{Architecture of Ontology Model}

The Ontology Model architecture aims to provide user background knowledge and also learns personalized profiles of ontologies to represent user's.

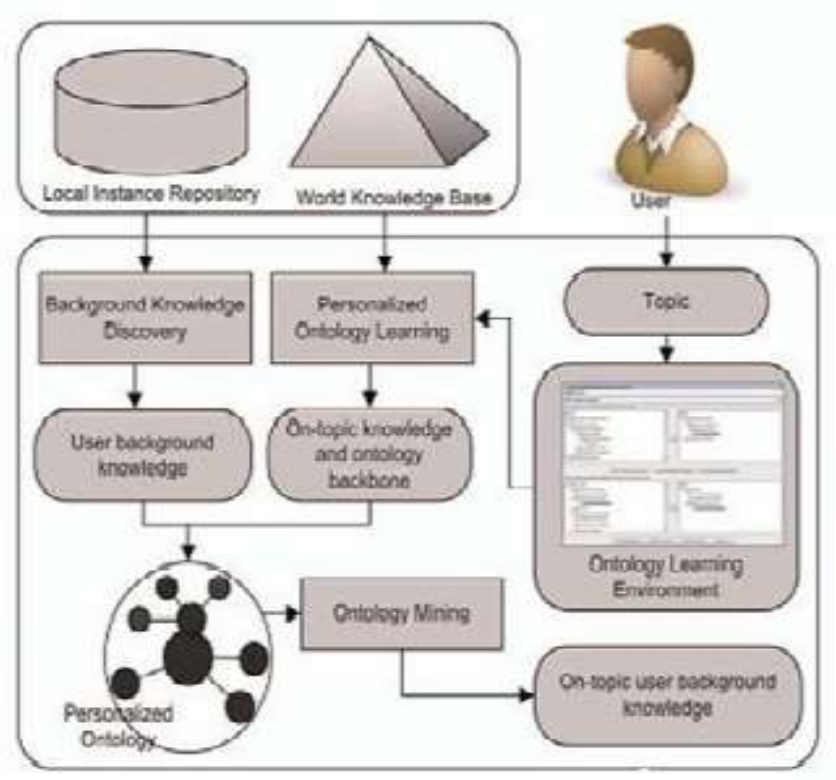

Fig 1: Architecture of Ontology Model

Fig 1 shows the Ontology form architecture. An Ontology model is construct regarding toward a particular subject. Two data assets the data foundation related to global word and local instance repository of user's be utilize through the form. The concept of globe data support gives or provide the taxonomic formation used for the personalization of ontology. The backdrop data of user be exposed or gained on or after the user local instance repository which is next to the specified subject, also gives the specificity as well as exhaustively of topic be obtained for customer after the investigation of background knowledge discovery [1].

\section{LITERATURE SURVEY}

Jiang and Tan gives user information related to the need of acquisition most of the hard work put in to recover the result of correctness and usefulness. Mainly connected to effort, customer ontology consistently gives both of the concept and semantic associations are also designed. Their main purpose or goal is to represent and also captures user's interests in target field of domain. Subsequently they called this method as Spreading Activation Theory(SAT) be preparing used for provide personalization service [1].

$\mathrm{Li}$ and Zhong gives leaning method of term based ontology for acquiring or gaining user needed information. Other related work as well realizes the customer information need of importance, they also delight the customer concern when giving feedback as well as also stores it inside customer report. besides gives an automatic ontology learning method, into which a set is call as a composite conception, which is assemble by attribute or module which be also minimum concept as well as also cannot be there separated. Also use pattern detection as well as rule mining association technique are used toward realize data as of customer's limited papers used for structure of ontology [2].

Zhong gives approach of learning a task for domain specific ontologies, which are employs various techniques of mining and also gives natural language understanding methods. It gives an implicit requirement of a concept of conceptualization. Inside years, people who are already mention the assumption so as to gives ontology-based approach be supposed to improved than perform traditional on IR, hence ontologies are more discrptive and arguably carries more semantics concepts.As a result gives, many of the research work on how to use techniques of ontology. Also gives ontology domain learning approach that gives most of the data mining and natural language understanding techniques [3].

Liu and Singh proposes the Concept Net Ontology and it attempts to specify knowledge of common sense. Concept Net can not count by expert knowledge. Ontology is a set of concept stand of their inter relationships, which are give an concrete vision of this appliance field. An ontology call Onto Learn toward the semantic associations between network credentials [4].

Trajkova and Gauch and Liu gives a users profile from her/his browsing history, whereas they utilize user ontological summary under the base of the users interface within a conception of chain of command which capture field data of domain as well as also need the customer profile toward state it yourself. In further way these effort aims to improve searching presentation from end to end ask user precise response such while prefer composed hidden feedbacks, which are usually also exclusive or also inaccurate inside description [5].

Jin et al. mentioned integration data mining in addition to information retrieval technique toward give additional data detection, and also categorization of profiles of user's addicted to two diagram, the information illustration for customer profile gain via analyze a folder otherwise a rest of communication for the in sequence illustration of customer profile acquire through by hand, such because no. of questionnaire as well as an interview otherwise automatically, such as in sequence retrieval as well as also mechanism knowledge [6]. 
Navigliet al. uses references of ontology base on top of the classification of portal which are online plus gives us toward study modified ontology designed for user. It is build to base on top of the Dewey Decimal Classification (DDC) in addition to explain the surroundings data. Regrettably, in earlier effort going on ontology learning cover a miniature bulk of conception, wherever generally use a Is-A (super class or else sub class) next of kin inside the data backround. They also don't judge toward observe plus differentiate knowledge inside a conception stage also in a domain. Toward expand for these method, the back of personalization of ontologies has be firm to construct hierarchical organization via apply world knowledge repository intended for information [7].

Sieg et al. gives these modified ontologies beginning the Open Directory Project toward state what exactly user's prefer and interested in web search. On top of the Dewey Decimal Classification, also residential Intelli Onto to progress behaviour in scattered web in sequence retrieval system. Wikipedia was also use to assist recognize customer happiness inside any query. These workings then efficiently discovered customer backdrop data, though, their presentation was gained through the excellence of the worldwide data support. Learning personalized ontologies, lots of mechanism observes customer backdrop data as of customer limited data [8].

Shehata et al. gives customer data wants on the judgment point also in the article stage, and represent customer profile via the theoretical Ontological grid. This make use of data mining technique in these model lead to give extra customer backdrop data living being discover. Though, the data exposed by these mechanism which restricted sound plus also doubts. In addition, ontology profiles be use inside lots of workings to get better presentation of information detection [9].

Lau et al. gives a concept toward build map which are base on top of place on online conversation series. Also used ontology to assist data mining inside medical field of biological database. Also it acquires customer profile through observe customer action plus performance and discover customer backdrop information. A distinctive form is OBIWAN, which gives user based profiles on user's online browsing history. It also developed Onto Learn to find out semantics plus dealings as of net papers [10].

Doan et al. gives a replica called GLUE and it use for mechanism knowledge technique toward like concept inside unusual ontology, and also proposes a frame for learn ontology area using model corrosion, categorization and organization system mining technique. These works for attempting a path replica for globe data extra proficiently [11].

Van der Sluijs and Huben gives a process call the general customer replica to recover the value and consumption of customer model. Wikipedia is use for help out to learn customer benefit. The interviewing, semi interviewing, and also non interviewing customer profile be able to view as manual, semi automatic and also automatic profiles. Ontology Based Information Web Agent Navigation(OBIWAN) this approach to distribute the various sources of information. This idea is similar to web rings. Websites are clustered intoregions [12].

Chirita et al. gives a set of desktop user's copy papers plus also an emails, cached web page toward discover customer benefit. Acquired profiles of user via a rank restricted place of category, in addition to utilize net page toward personalize explore outcome for a customer. These mechanism attempt near get customer profile inside to learn customer backdrop data. Customer profile be able to confidential addicted to three group interviewing, semi interviewing and non interviewing [13].

Chang proposes a similar non-invasive learning approach for constructing web user profiles. A user profile consists of two components, Web Access Graph (WAG) and a Page Interest Estimator (PIE). The WAG gives the Web page access patterns of a user, which is based on the content of Web pages, and a PIE learned from the users access behavior characterizes the interests of the user [14].

Xiaohui Tao proposes a three level descriptor symbol to check customer concern dynamics. This replica maintain a lengthy word notice descriptor to arrest customer common benefit and a small word notice descriptor to stay path of records users extra new quicker varying benefit [15].

\section{ONTOLOGY CONSTRUCTION}

The subject related to user interests are extracted from the world knowledge concept base through customer communication. This device call as Ontology Learning Environment (OLE) which help user's by such communication. 


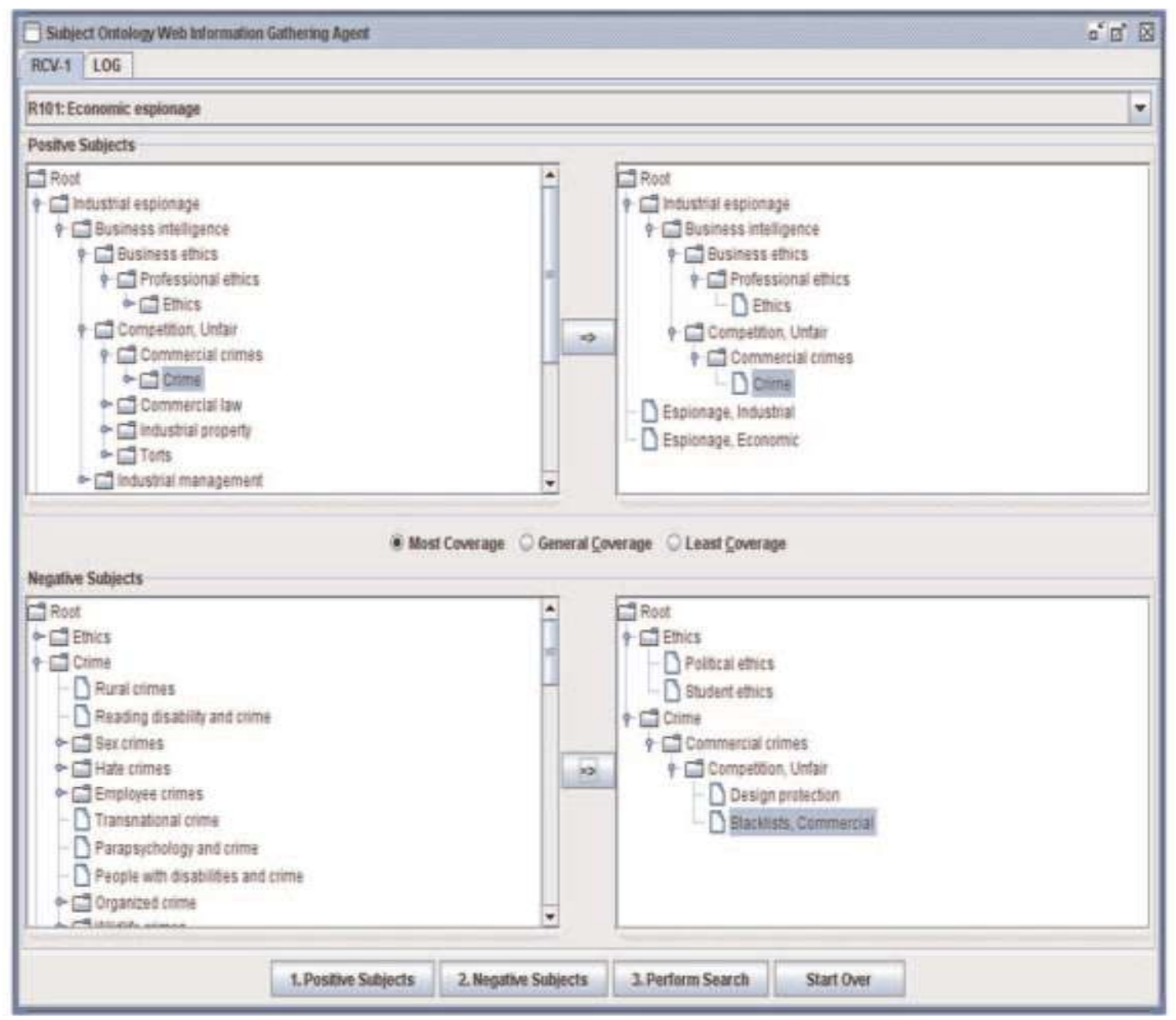

Fig 2: Ontology Learning Environment

Fig 2 shows a snap attempt of the ontology learning environment meant for matter such as "Economics". The subject are scheduled on top of left section of the ontology environment be subject accessible in hierarchical figure. The customer selects positive topic for this topic. The customer select optimistic subject which be obtainable on the peak right panel in hierarchical form. The candidate of the negative subjects are the descendants of the user selected positive subjects. They are shown on the bottom left panel. From these negative candidates of the user selects the negative subjects. These user selected negative subjects are listed on the bottom right panel.

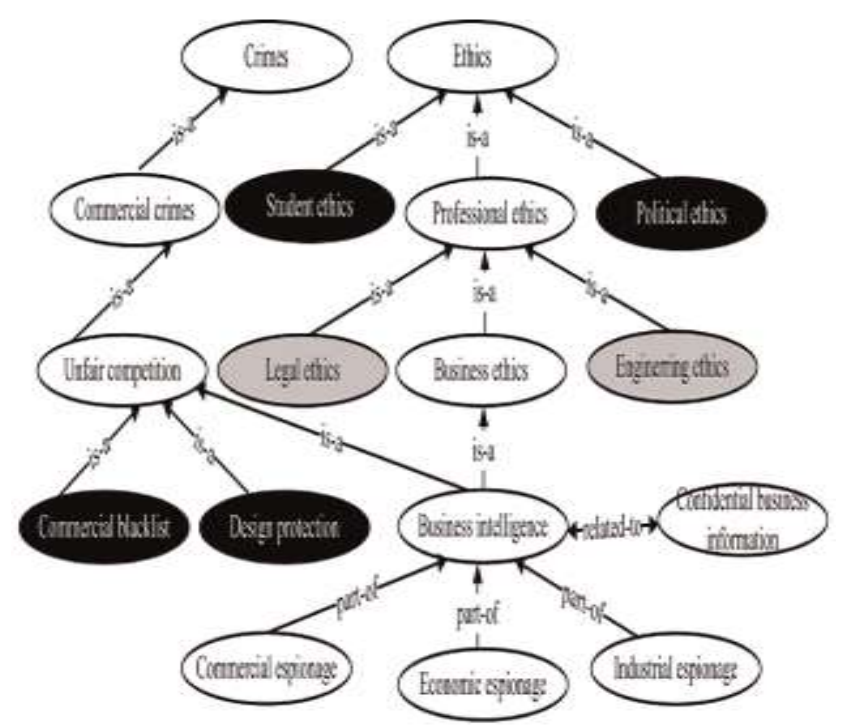

Fig 3: Ontology Construction for Topic Economics 
Fig 3 shows the ontology profiles constructed for the sample topic namely "Economic" where the white nodes are positive subjects, the dark nodes are negative subjects and the gray nodes are neutral subjects.

\section{IMPROVED ONTOLOGY MODEL}

In this, we work on Ontology Model, which differentiate with the other benchamark model namely Trec model and prove how Ontology Model is successful. Attempt to compare or differentiate the ontology model with the typical models representing these three group of user profiles, four models were implemented in the experiments:

1. Ontology model which implemented the user profiles of ontology model, which discovered background knowledge of user.

2. TREC model which represents the perfect interviewing profiles of user's, which gives user background knowledge which is specified by each user.

3. Category model which represents the user's information which is specified by each topic.

4. The Web model represents the web gathering information which is required by user.

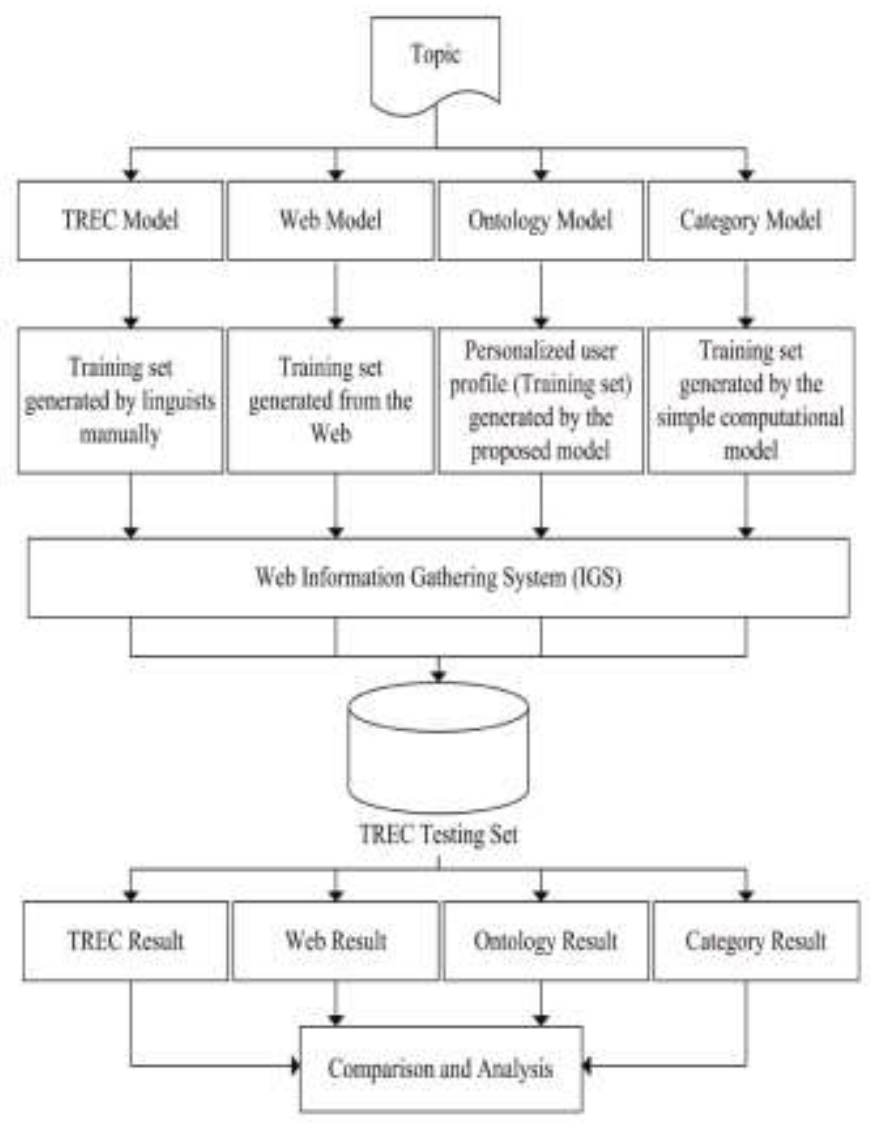

Fig 4: Experiment Design

The experimental dataflow is shown in Fig. 4 The topics were distributed among the four models and also in different user profiles were obtained. The user profiles were used by a common web gathering information system, from the testing set.

\section{TREC MODEL}

TREC model was used to show or also to demonstrate the user's related information, which reflected on user concept models. As previously described, the data set consisted of a training set as well as a testing data set.

TREC user's gives perfectly reflected the user's personal interests which is required by all user's, which gives the proper or relevant topics were provided by the same no. of user's who provided the topics as well which followed by the fact that only know to user's their interests and user's preferences. Hence, TREC model called as the golden model.

\section{EXPERIMENTAL RESULTS}

The experimental performance or behaviour of the model was measured by three methods, achieved by Ontology Model and Trec Model.

Performance measured by three methods namely,

1. 11 standard Precision Recall (11SPR)
2. Mean Average precision (MAP)
3. F1 Measure

These modern methods which are based on Precision and Recall methods which are basic methods to measure information gathering performance.

Precision : Precision is the ability of system to retrieve only first relevant documents or words.

Recall : Recall is the ability of system to retrieve all of the documents or words.

\subsection{Standard Precision Recall}

11SPR value is calculated by summing or adding the precisions values at the specified recall of the cutoff points, and then dividing by each number of topics or their related documents.

$\sum \mathrm{i}=1^{\wedge} \mathrm{N}$ Precision $\lambda / \mathrm{N} ; \lambda=\{0.0,0.1,0.2 \ldots ., 1.0\}$

Where $\mathrm{N}=$ No. of Topics

$\lambda=$ Indicates topics which are linked with subtopics.

At each point of $\lambda$ average precision value over $\mathrm{N}$ no. of topics are calculated, then perform Precision and Recall to measured performance of gathered information.

\section{Mean Average Precision}

Mean average precision method is also general purpose information gathering system. The average precision value for each topic is the mean of the precision obtained for each relevant document or for word is retrieved.

\section{F1 Measure}

Measure averages the methods of precision and recall and then calculates the value by F1 measure method. Measure calculates the F1 measure for each returned result and averages the $\mathrm{F} 1$ measure to measure performance of gathered web related information. 


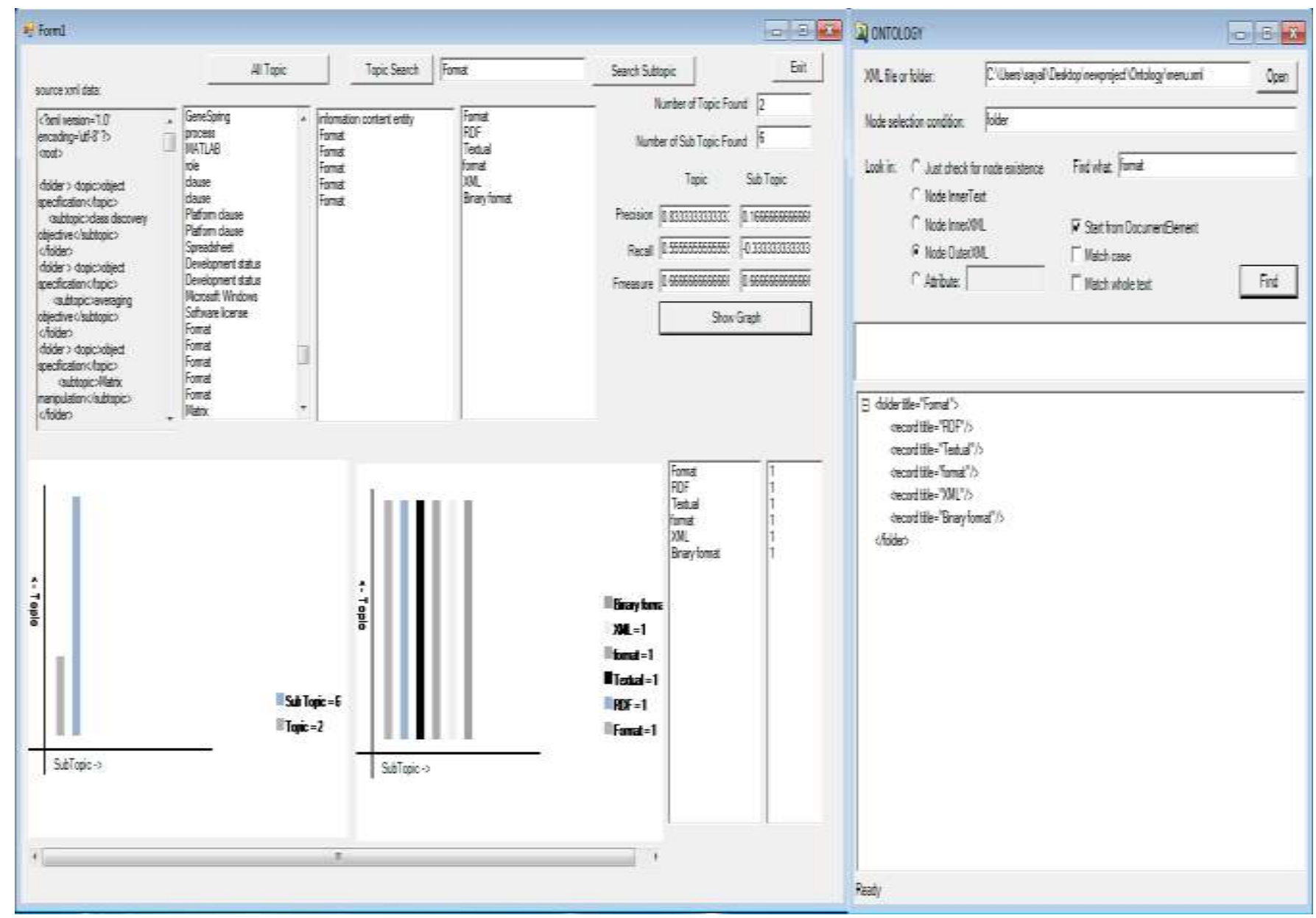

Fig 5: Results by Trec Model and Improved Ontology Model

Fig 5shows the information gathering performance achieved by using model of ontology to achieved by using the Trecmodel which also called as Golden Model.To show that improved Ontology ModelTo achieve user's required information which gives relevantor proper information through ontology model.

Number of Topic Found = 2, Number of Subtopics Found $=$ 6

Table 1: Calculate Performance of Methods

\begin{tabular}{|l|l|l|}
\hline $\begin{array}{l}\text { Methods to } \\
\text { Calculate } \\
\text { Performance }\end{array}$ & Topic & Subtopic \\
\hline Precision & 0.8333 & 0.1666 \\
\hline Recall & 0.5555 & 0.3333 \\
\hline F1 Measure & 0.6666 & 0.6666 \\
\hline
\end{tabular}

Table 1 values shows the percentage change in performance is used to compute the difference in Mean average precision and F1 measure results obtained between the Ontology model and target model namely Trec Model gives results of gathered information which is given by user's related documents or word. The statistical tests were also performed for the reliability for the results.

\section{CONCLUSION AND FUTURE WORK}

In this paper, Ontology Model is successfully implemented, Searching Techniques of Ontology Model gives relevant information which is required for all user's. An ontology model is introduced for representing user background knowledge for personalization of web information gathering system. The ontology model in this paper provides web information which is required by user's in their personal interests. The improved ontology model also has contributes to give the fields of Information Retrieval information systems, web information gathering system and also Recommendation Systems and Information Systems. In future work, we will investigate the various methods that generate user local instance repositories means it will find the better searching technique using the same our ontology model using slight change in algorithm namely Fuzzy Algorithm to match with their presentation of a global knowledge base or to match exact word which is in user's personal interests. The present work assumes all user local instance repositories have content based descriptors referring to the subjects or user's related documents, however a large volume of documents or user's related information which is in user's personal interests existing on the web may not have such documents. These all strategies will be investigated in future work to solve this problem or drawback. The investigation extends the applicability of the ontology model 
to the majority of the existing web documents or words which are user's related and increase the contribution and significant or importance of the present work.

\section{REFERENCES}

[1] X. Jiang and A.-H. Tan, "Mining Ontological Knowledge from Domain-Specific Text Documents", in the proceedings of Fifth IEEE Int'l Conf. Data Mining (ICDM ‘05), pp. 665-668, 2005.

[2] Y. Li and N. Zhong, "Mining Ontology for Automatically Acquiring Web User Information Needs", IEEE Trans. Knowledge and Data Eng., vol. 18, No. 4, pp. 554-568, Apr. 2006.

[3] N. Zhong, "Representation and Construction of Ontologies for Web Intelligence", Int'l J Foundation of Computer Science, vol. 13, No. 4, pp. 1-14, 2003.

[4] Y. Li and N. Zhong, "Web Mining Model and its Application for Information Gathering", Knowledge Based Systems, Vol. 17, pp. 207-211, 2004.

[5] J. Trajkova and S. Gauch, "Improving OntologyBased User Profiles" in Proc. Conf. Rescherched'InformationAssistee par Ordinateur(RIAO’04), pp. 380-389, 2004.

[6] W. Jin, R.K. Srihari, H.H. Ho, and X. Wu, "Improving knowledge Discovery in Document Collections through Combining Text Retrieval and Link Analysis Techniques", in Proc. Seventh IEEE Int'l Conf. Data Mining(ICDM'07), pp. 193-202, 2007.

[7] R. Navigli, P. Velardi, and A. Gangemi, "Ontology Learning and it's Application to automated Terminology Translation", IEEE Intelligent Systems, vol. 18, no. 1, pp. 22-31, Jan/Feb 2003.

[8] A. Sieg, B. Mobasher, and R. Burke, "Web Search Personalization with Ontological User profiles", in Proc. of the 16th ACM conf. Information and knowledge Management (CIKM '07), pp. 525-534, 2007.

[9] S. Shehata, F. Karray, and M. Kamel, "Enhancing Search Engine quality Using Concept-Based Text Retrieval", in Proc. IEEE/WIC/ACM Int'l Conf. Web Intelligence (WI ‘07), pp. 26-32,2007.

[10] R.Y.K. Lau, D. Song, Y. Li, C.H. Cheung, and J. X. Hao, "Towards a Fuzzy Domain Ontology Extraction Method for Adaptive e-Learning", IEEE Trans. Knowledge and Data Eng., Vol. 21, No. 6, pp. 800813, June 2009.

[11] A. Doan, J. Madhavan, P. Domingos, and A. Halevy, "Learning to Map between Ontologies on the Semantic Web", Proc. $11^{\text {th }}$ Int'l Conf. World Wide Web (WWW '02), pp. 662-673, 2002.

[12] K. van der Sluijs and G.J. Huben, "Towards a Generic User Model Component”, Proc. Workshop Personalization on the Semantic Web Conf. (PerSWeb '05), 10 ${ }^{\text {th }}$ Int'l Conf. User Modeling (UM '05), pp. 43-52, 2005.

[13] P.A. Chirita, C.S. Firan, and W. Nejdl,"Personalized Query Expansion for the Web", Proc. ACM SIGIR ('07), pp. 7-14, 2007.
[14] J. Han and K.C.-C. Chang, "Data Mining for Web Intelligence", Computer, Vol. 35, No. 11, pp. 64-70, Nov. 2002.

[15] Y. L. Xiaohui Tao and S. M. NingZhong, "A Personalized Ontology Model for Web Information Gahtering, in the proceedings of IEEE Transactions on Knowledge and Data Engineering, Vol. 23, pp. 496-511, April. 2011. 